\title{
REVIEW OF ASSEMBLY SEQUENCE PLANNING METHODS IN TERMS OF THEIR APPLICABILITY IN SHIPBUILDING PROCESSES
}

\author{
Michał Taraska ${ }^{1}$ \\ Remigiusz Iwańkowicz ${ }^{2}$ \\ Tomasz Urbański ${ }^{1}$ \\ Tadeusz Graczyk ${ }^{1}$ \\ ${ }^{1}$ West Pomeranian University of Technology in Szczecin, Poland \\ ${ }^{2}$ Maritime University of Szczecin, Faculty of Transport Engineering and Economics, Poland
}

\begin{abstract}
The article characterises the sea-going vessel hull assembly processes and then reviews the existing assembly methods of mechanisms and welded ship structures. Classification of these methods is done with respect to selected criteria of their applicability to hull assembly. Selected methods are used for calculations performed on a model structure and exemplary database. The analysed aspects include the performance of calculation algorithms and the quality of the obtained solutions. Particular attention is paid to the need for reduction of experts' participation in the planning process due to strong search space explosion effect. The performed analyses have enabled the authors to formulate assumptions for models which would be applicable in real assembly planning in shipyards, as well as to indicate areas of further research which would make it possible to better consider the specificity of production of large-size welded structures.
\end{abstract}

Keywords: assembly sequence planning, ship hull, welded structure, method review, shipyard

\section{INTRODUCTION}

An important role of assembly sequence planning (ASP) automatization methods in the shipbuilding industry is frequently stressed in contemporary literature [1] [2] [3]. The need for ship hull assembly sequence planning methods was initially noticed as early as in 1979 [4]. Then, numerous authors have indicated the assembly as most important moment in the ship hull building cycle [5] [6] [7]. Adapting ASP methods used for assembly of mechanisms to the shipbuilding industry is not always possible due to its specificity and the size of the built structures. The biggest obstacle is the number of parts composing the ship hull, and the resultant size of the space of possible assembly sequences. This size can be assessed using the Robinson formula to calculate the number of different directed acyclic graphs (DAG) [8] [9]:

$$
G(n)=\sum_{k=1}^{n}(-1)^{k-1} \cdot\left(\begin{array}{l}
n \\
k
\end{array}\right) \cdot 2^{k \cdot(n-k)} \cdot G(n-k), \quad G(0)=1
$$

where $n$ is the number of graph nodes.

The numerical amount of the search space expressed in the above way increases in a very dynamic way. Formula (1) says that for 10 nodes, more than $4 \cdot 10^{18}$ different graphs can be generated.

For hulls of typical merchant ships, the number of liaisons can exceed hundreds of thousands, and consequently, complete search of the space of acceptable assembly sequence solutions is impossible. This is well illustrated by an attempt 
to use the method proposed in [10] for a simple ship structure. The algorithm proposed in that article develops and simplifies the method which is considered classical in the field of assembly of mechanisms [11]. An exemplary solution for a ship structure which bases on that method is shown in Chapter 3.2.

Basic division of ASP methods refers to the purpose of their use. Here, a distinction is made between methods which search for an acceptable solution and those which search for optimum. In the former group, complete search of the space of solutions is frequently applied to extract the acceptable solution based on selected criteria. This is an important methodology for sequence planning of mechanism assembly, therefore it has been developed from very beginning of the appearance of this problem. Attempts to expand the problem by adding new issues most often lead to the optimisation model. Here, a possible direction of development can be taking into account criteria which minimise the number of adjustment operations or the number of changes of the equipment used in the process.

Hull assembly is less sensitive to geometric constraints than typical mechanisms analysed in the literature. The hull is a so-called sparse structure, which means that its parts are distributed in the space in a more dispersed way that elements of mechanisms. The integrity of hull structure is ensured by permanent joints - welds.

The next important aspect of each individual method is the level of experts' involvement in the calculation process. Their assistance cannot be totally eliminated, as they are needed for, among other tasks, assuming weights of optimisation criteria, selecting data introduced to the code at the beginning of the calculation process, and assessing the obtained results. However, a number of methods can be named which base on wider interaction with experts, who are expected to answer a series of questions, or perform cluster analysis of the database.

The aim of this article is to analyse the group of existing methods, concerning both mechanism assembly planning and those clearly dedicated to shipbuilding, with respect to their applicability to ship hull assembly planning. These methods have been classified with respect to their basic and repeating features, and their applicability to hull assembly has been assessed. Selected methods were used in calculations performed on a model structure.

\section{LITERATURE REVIEW}

With his Ph.D. thesis in 1984, Bourjault [11] initiated the development of ASP methods for mechanisms. These methods based on complete search of the set of acceptable solutions and consisted in checking whether the execution of certain liaison leads to the state from which the final structure can be obtained. This approach generated trees of all possible assembly plans for a given structure [10] [11]. In [12], the author proposes the use of "And/Or" graphs for backward analysis of mechanism assembly sequences. The next publication of the same authors [13] illustrates the flexibility of "And/Or" graphs and their potential for easy modifications to obtain new functionalities. However, both works reveal dynamic increase of the number of possible solutions as a result of the increased complexity of the analysed task. Consequently, a possibility of their adaptation to ship hull assembly planning problems seems to be marginal.

The complete search-based methods lose dramatically their efficiency when the complexity of the structure increases. In [14], the authors have remarked that taking into consideration geometric constraints of structure assembly is necessary but insufficient for correct assessment of solution acceptability. Four criteria were proposed for assessing the generated sequence.

In the case of ship structures, standard geometric constraints are to be complemented with welding positions. This was taken into account in [5], which proposed the ship hull welding planning assistance system. The presented method was not fully compatible with ASP solutions, it was merely an idea to be used as an assembly planning assistance module. The problem of welding operation sequence planning is frequently considered as a separate issue, solved using intelligent methods, such as genetic algorithms or annealing, or the finite element method [15].

In [16], the authors have remarked that the 3D modelling and simulation-based planning process reveals good performance in terms of work strategy planning. The authors propose the ACIM (advanced computer integrated manufacturing system) type algorithm, but describe its essence in a sketchy way, which makes assessing its efficiency and reconstruction of the computation process impossible.

In [17], the authors were the first to suggest the use of genetic algorithms (GA) as a tool for finding optimal and semi-optimal assembly plans. However, the efficiency of the described algorithm is difficult to assess, as the final result is strongly affected by the initial population of proposed structures, prepared by an expert. The idea to use GA in assembly planning processes was developed in [18], the author of which proposes an adaptive genetic algorithm. This algorithm is characterised by varying weights of appearance of genetic operators (mutation and crossing), which in classical GA are constant for all populations. The author makes distinction between two types of structure constraints: physical and geometric. Meeting all geometric constraints by the proposed assembly plan was identified as the necessary condition for considering it an acceptable solution. Physical constraints concerning such aspects as available equipment, or assembly difficulties and cost, were used as optimisation criteria for the proposed algorithm. The method described in the paper was tested on selected mechanical structures.

In recent years, great interest of shipbuilding industry has been focused on CBR (case-based reasoning) methods as a possible ASP assistance tool. In [19], the authors propose the CBR-CAPP (CBR-computer aided process planning) algorithm for automatic assembly planning of small ship blocks. However, the solution proposed by the authors is difficult to implement in other cases that that discussed in 
the article, as its correct operation requires a huge database of similar structures, worked out by experts, which is a typical drawback of CBR methods. As a result, for structures which require special assembly principles the method is of little or no use. Using CBR methods reduces participation of experts in the assembly planning process, but their active participation is still required when preparing the database. A positive aspect here is that consecutive solutions obtained using this method are introduced to the database and increase it, which leads to the decrease of general participation of experts in the assembly planning and tool preparation processes.

In [20], the author proposes combining CBR with GA to obtain a tool which enables generating assembly plans for marine structures. The proposed GA makes use of geometric constraints as one of optimisation criteria, and the criterion which minimises changes of component classes during serial assembly as the other criterion. A weak point of the method is the need for extensive database of similar structures, like in all problems making use of CBR methods. For untypical or new assembly units, the genetic algorithms alone may turn our insufficient for generating a satisfying assembly plan. Moreover, an important role in the proposed assembly planning methodology based on the database of similar cases is played be an expert, as this methodology requires unambiguous classification of all components of the analysed structure, which in the case of sea-going hulls is not always evident. All this makes that the reduction of experts' participation in the assembly process, which is typical for intelligent algorithms, is hardly perceivable in the above case.

\section{ANALYSING PROPOSED METHODS WITH RESPECT TO THEIR APPLICABILITY TO HULL ASSEMBLY.}

\section{DESCRIPTION OF EXEMPLARY STRUCTURE}

To perform the analysis of selected methods in terms of their applicability to practical hull assembly planning, a simplified exemplary structure was defined which consisted of five parts and six liaisons between them. This structure is given the number 0 and name S0, while its parts get consecutive numbers with hyphen: $0-1$ - plate, $0-2$ - web of T-frame, 0-3 - flange of T-frame, and 0-4, 0-5 - ribs.

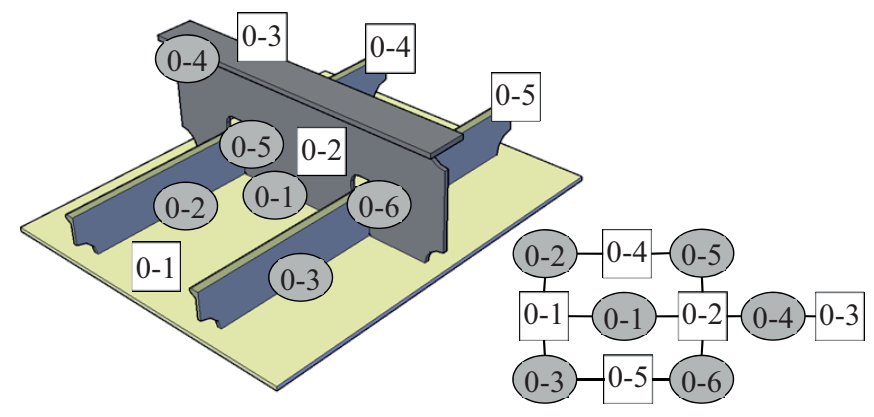

Fig. 1. Exemplary assembly unit So
All liaisons between parts are made as fillet welds. Fig. 1 shows numbers of parts (in squares) and the so-called liaison graph with number of liaisons (in circles).

The structure can be assembled in a number of different ways. Each sequence includes intermediate stages of structure state (subassemblies) which consist of two, three, or four parts. Fig. 6 shows all possible variants, including those which are irrational from the point of view of traditional assembly planning methods used in shipyards.

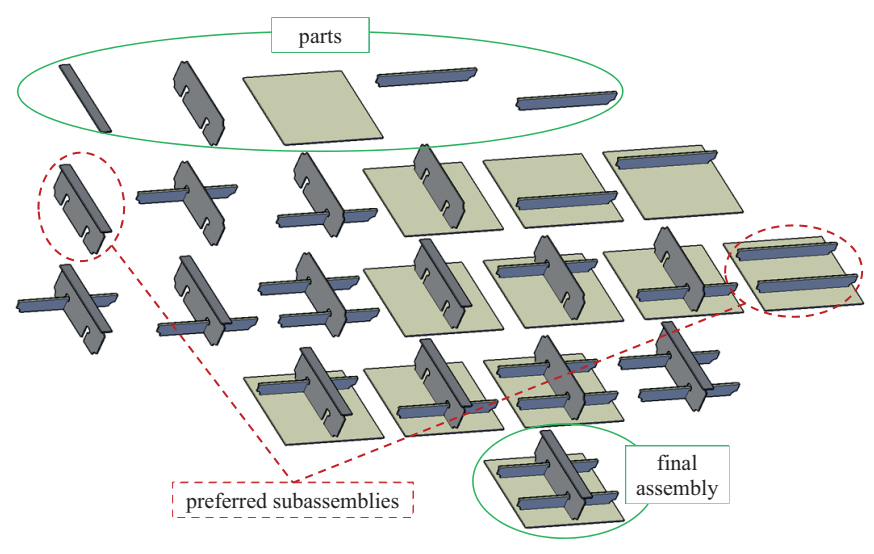

Fig. 2. Possible variants of intermediate stages during assembly process of unit SO

The required assembly sequence for the unit S0 is initial subassembly of the T-frame and welding the ribs to the plate. These two operations can be done simultaneously. The next operation should be connecting the obtained subassemblies together. It should be kept in mind, however, that the correctly prepared process should define not only the order of connecting structure parts, but also the order of making consecutive welded joints. In the analysed case, the T-frame can be connected with the stiffened plate either by making liaisons between the frame and the ribs and then between the frame and the plate, or in the reverse order. Both variants are completely invisible in the plan oriented only on structure parts.

In its further part, the article analyses abilities of different ASP methods to generate assembly sequences which, in authors' opinion, can be of practical applicability or reveal potential for development.

\section{DETERMINING CRITERIA FOR BEST METHOD SELECTION}

To assess which method described in the literature review is most useful for ship hull assembly planning, first the desired features of the method are to be determined. Then, these features will be used as criteria for selection of best solutions. For this purpose, the prepared exemplary structure was analysed with the aid of the classical method developed by Bourjault [11] and improved by De Fazio and Whitney [10]. That method bases on numbering liaisons in the structure and formulating a series of questions concerning relations between these liaisons. Further in the paper, this method is 
referred to as the Q\&A (questions \& answers) method. The above analysis will make it possible to identify basic problems resulting from the specificity of hull structures and thus determine which features of searched methods will allow us to avoid or minimise these problems.

In the analysed Q\&A method, answers to the questions are always given by an expert - process engineer, who has knowledge on the course of assembly processes and on features of the assembled structure. The Q\&A method in version given by De Fazio and Whitney [10] consists in two questions concerning each i-th liaison:

- Which liaison must be done prior to doing liaison $i$ ?

- Which liaisons must be left to be done after doing liaison $i$ ?

The answers to these questions make the basis for defining precedence relations between liaisons. Experts' answers can take into account arbitrary factors, which in the simplest version can refer to the geometry of elements and physical possibilities of placing them in a right place during the assembly. The expert can answer these simple questions fast, therefore the procedure consisting of tens or even hundreds of questions can be easily applied in practice.

Let us consider a simplified exemplary structure (Fig. 1). If we take into account only geometric constraints, then for each liaison identical answers: "none of the remaining liaisons" are to be given to both questions. This results from the fact that the parts do not create geometrical conflicts and can be added to the structure in an arbitrary order. The same refers to the order of doing liaisons. For instance, after adding T-bar to the plate earlier connected with bulb stiffeners, liaisons between the T-bar and the plate, and between the T-bar and the stiffeners are to be done. Theoretically, these three liaisons can be done in an arbitrary order and in this situation the Q\&A method based on geometric constraints fails, as it does not reduce the set of acceptable assembly sequences.

Indeed, along with geometric constrains, the Q\&A method can take into account also other aspects. The expert providing the answers can consider typical hull related problems, such as welding deformations, assembly positions, and/or ergonomics and safety of welders' work.

For instance, let us assume that we want to do welding of the greatest possible number of liaisons:

1) in unforced positions,

2) steadily along the entire length.

Let us perform the analyse with the procedure given by De Fazio and Whitney [10]. For consecutive liaisons numbered as $i=1, \ldots, 6$ two questions are to be answered:

Q1 - What Liaisons Must be Done Prior to Doing Liaison 0-i?

Answers:

$i=1,2,3,4$ : none of the liaisons must be done prior to doing these liaisons,

$i=5,6$ : doing these liaisons in unforced position requires placing the bulb profile on its side. This operation will not require special technological supports, provided that T-bar and stiffener liaisons with the plate are done prior to them:

$$
\{0-1,0-2\} \rightarrow 0-5
$$

$$
\{0-1,0-3\} \rightarrow 0-6
$$

Q2 - What Liaisons Must be Left to be Done After Doing Liaison $0-i$ ?

Answers:

$i=1$ : none of the liaisons must be done after connecting the web of the T-bar with the plate,

$i=2,3$ : if liaisons $0-2$ and $0-3$ are to be welded steadily along the entire length, then liaisons $0-1,0-5$ and $0-6$ must be done later:

$$
\begin{aligned}
& 0-2 \rightarrow\{0-1,0-5,0-6\} \\
& 0-3 \rightarrow\{0-1,0-5,0-6\}
\end{aligned}
$$

$i=4$ : if liaison $0-4$ is to be welded in unforced position, then liaisons $0-1,0-5$ and $0-6$ must be done later:

$$
0-4 \rightarrow\{0-1,0-5,0-6\}
$$

$i=5,6$ : none of the liaisons must be done after doing liaisons 0-5 and 0-6.

After completing all answers, a graph of sequences can be created (Fig. 3-a). Nodes in this graph represent liaisons, while arcs correspond to precedence relations. This is a directed acyclic graph.
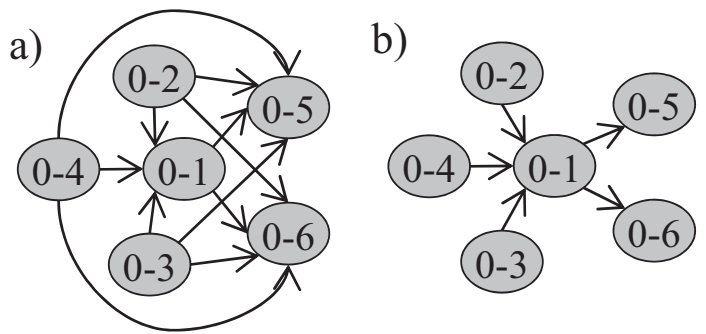

Fig. 3. Solution obtained by the QઐA method

It is noteworthy that the sequence obtained using the Q\&A method incudes surplus precedence relations, which can be removed. For instance, the precedence of action 0-4 over action 0-5 is a transitive closure of the precedence chain $0-4 \rightarrow 0-1 \rightarrow 0-5$. After removing all surplus precedence relations, we obtain a so-called minimal sequence graph (Fig. 3-b).

In the case of extensive technological analysis of each question in the Q\&A method, the time needed to give the answers becomes a problem. Moreover, methods which base on experts' assessment and are dedicated to mechanisms are difficult to adapt to the shipbuilding environment, due to wide variety of structures. Each structural type reacts to exothermic processes in a different way, as well as has different ergonomics and problematics of work. Adapting those methods leads to large experts' labour intensity and requires involving FEM type calculations, or even experimental methods on physical objects. Hence the conclusion that the searched methods should:

- be dedicated to ship hulls and marine structures, 
- take into account additional assembly constraints, along with the geometry,

- minimise experts' participation in the assessment and analysis process by applying intelligent and self-learning methods,

- be applicable and replicable for diversified structures.

The above features were recognised as criteria for selecting best methods from the set discussed in the literature review.

\section{SELECTING BEST METHOD FROM ANALYSED SET BASED ON SELECTED CRITERIA}

The methods discussed in the literature review were analysed with respect to the required features of the ship hull assembly planning method presented in Chapter 3.2. Table 1 collates results of this analysis. Consecutive columns are to be interpreted in the following way:

1. This column contains the information about the authors of the method, numbers in square brackets refer to the bibliography number of the article in which the method is described.

2. (I) Does the method description allow its calculation procedure to be reproduced?

3. (II) Is the method dedicated to ship hull assembly planning?

4. (III) Does the method take into consideration geometric constraints of assembly?

5. (IV) Does the method take into consideration other, additional assembly constraints?

6. (V) Does the method allow experts' participation to be reduced in the assembly planning process?

Tab 1. Results of applicability analysis of assembly planning methods

\begin{tabular}{|l|c|c|c|c|c|}
\hline \multicolumn{1}{|c|}{1} & 2 & 3 & 4 & 5 & 6 \\
\hline Method & I & II & III & IV & V \\
\hline Bourjault (1984) [11] & Yes & No & No & No & No \\
\hline De Fazio i Whitney (1987) [10] & Yes & No & No & No & No \\
\hline de Mello i Sanderson (1986) [12] & Yes & No & Yes & No & No \\
\hline de Mello i Sanderson (1988) [13] & Yes & No & Yes & No & No \\
\hline Eng et al. (1999) [14] & Yes & No & Yes & Yes & Yes \\
\hline Cho et al. (1999) [5] & No & Yes & No & Yes & - \\
\hline Sasaki et al. (2003) [16] & No & Yes & Yes & No & Yes \\
\hline Bonneville et al. (1995) [17] & Yes & No & No & Yes & No \\
\hline Chen i Liu (2001) [18] & Yes & No & Yes & Yes & - \\
\hline Seo et al. (2007) [19] & Yes & Yes & Yes & Yes & Yes \\
\hline Qu et al. (2013) [20] & Yes & Yes & Yes & Yes & No \\
\hline Zhong et al. (2013) [21] & Yes & Yes & Yes & Yes & No \\
\hline
\end{tabular}

For two methods, their effect on reducing experts' participation in the assembly planning process could not be assessed. In the case of the solution proposed in [5], this results from insufficient data in the article and the resultant impossibility to reproduce the calculation process needed to assess the scale of the required experts' participation. The article [18], in turn, is not dedicated to ship solutions and its adaptation to this area could dramatically worsen the scale of reduction of experts' work obtained for mechanism assembly planning. That is why a decision was made to assign no values to these two methods in this field.

From among analysed methods, four methods have outstanding results which are, consecutively, methods: [14], [19], [20] and [21]. However, the work [14] is not dedicated to hull assembly, consequently its implementation in this area would be very time and effort consuming. Therefore, a decision was made to omit this method in further considerations. For the remaining methods, numerical calculations were performed on the exemplary structure presented in Chapter 3.1 to identify strengths and weaknesses of each individual method.

\section{APPLYING SELECTED METHODS IN HULL STRUCTURE ASSEMBLY PLANNING}

\section{ACTIVITIES-ORIENTED ASSEMBLY PLANNING ON THE BASE OF CASES}

The method proposed by Seo, et al. [19] makes use of the database of similar structures and the CBR procedure which comprises typical stages:

- database search for cases similar to the new structure,

- determining a new sequence based on similar cases,

- verification of the solution,

- database updating.

The structures stored in the database are referred to as cases, while the structure for which the assembly sequence is to be created bears the name of a problem. Each structure is characterised by:

- set of parts and assigned classes: plate, rib, girder),

- set of liaisons and assigned classes: fillet, butt, through),

- set of constraints: precedence relations between liaisons, assignment of liaisons to common group, alternativity of liaisons,

- set of assembly sequences - determined only for cases - each sequence is serial in nature, i.e. is a permutation of structure liaison numbers.

A new sequence generated for structure-problem is serial in nature. This is a serious limitation of the method. For instance, a result of serial sequencing can be blockage ofpossible subassembly of all T-frames before their connecting with the rest of the structure. This problem can appear when the structure comprises more than one girder. For the first girder, a good solution could be placing its flange at the beginning of the sequence and its web in the second place.

The authors have introduced graphical way of representation of parts and liaisons in the liaison graph. This approach seems questionable in the case of larger structures, with greater structural diversity. In this case both readability of the graph, and the process of part classification itself, to be done by an expert, can be a problem.

To illustrate the essence of the method, two structurecases are considered (Fig. 4), similar to the structure-problem 
(Fig. 1) for which a new sequence is to be created. In these exemplary considerations we neglect the problem of search for similar cases to simplify the analysis. The inference will be performed based on each case separately, and then an attempt will be made to include the information about these two cases from the database.
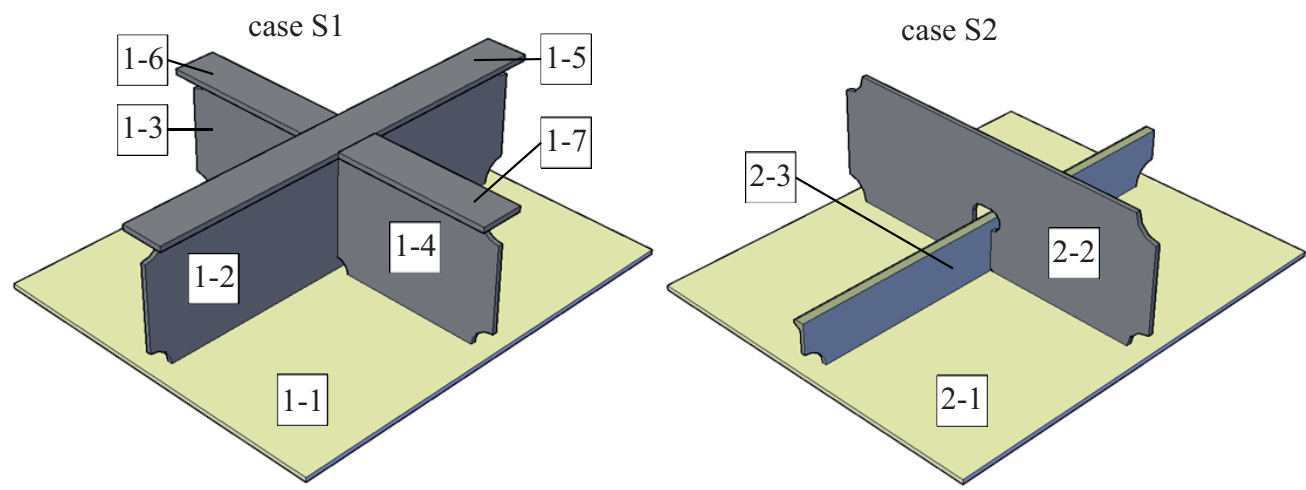

Fig. 4. Assembly units - 'cases' stored in exemplary database

Seo et al. proposed 3 classes of liaisons: fillet, butt, and through, and 3 classes of parts: plate, rib, and girder. Since the analysed structures comprise also other parts, additional classes of parts are assumed, which are: web and flange of T-frame.

The assembly sequences stored in the database according to the model by Seo et al., are the following:

- case S1: fillet(1-2,1-5), fillet(1-3,1-6), fillet(1-4,1-7), fillet(1-5 1-2, 1-1), fillet(1-7 1-4, 1-1 1-2 1-5), fillet(1-7 1-4, 1-2 1-5 1-1), butt(1-4 1-7, 1-5 1-2 1-1), fillet (1-6 1-3, 1-1 1-2 1-5 1-4 1-7), fillet (1-6 1-3, 1-2 1-5 1-1 1-4 1-7), butt (1-3 1-6, 1-5 1-2 1-1 1-4 1-7),

- case S2: fillet(2-1, 2-3), through(2-1 2-3, 2-2), fillet(2-2, 2-1 2-3).

The method of sequence recording proposed in [19] enables parallel assembly planning. This is possible due to planning oriented on activities, i.e. liaison welding operations. It should be kept in mind, however, that parallel doing of liaisons in the discussed model is only possible to a limited extent.

After introducing the classification of parts, we obtain a set of rules to be used when defining the assembly sequence for the new structure, numbered 0 . Here, a typical problem for CBR methods can be observed, which is strong dependence of the obtained solution on the quality of the database and the adopted way of case description. Based on case S1, we obtain the rule in which T-frames are prefabricated before welding to the plate. This is insufficient for planning the assembly sequence for the entire structure 0 . On the other hand, the rule resulting from case $\mathrm{S} 2$ says that ribs should be welded to the plate before their connecting with the girder. Applying this rule to the structure $\mathrm{S} 0$ does not provide a comprehensive solution to the structure assembly planning either.

\section{PARTS-ORIENTED ASSEMBLY PLANNING ON THE BASE OF CASES}

The method proposed by Qu et al. [20] enables to generate assembly sequences for a new structure based on cases stored in the database. Many similarities can be found between this method and that proposed by Seo et al. [19], the basic of which are the use of CBR procedure and serial nature of sequences.

What is characteristic, the method focuses on setting the order in which parts are to be connected. The planned sequence does not provide opportunities for planning the welding sequence. The parts-oriented approach has been developed for mechanism assembly, but its application to hull structures seems unjustified.

An exemplary analysis is performed based on the same case base (Fig. 4) and the same structure-problem, labelled as S0 (Fig. 1). For the case S1, the sequence of part liaisons is: (1-5, $1-2,1-1,1-3,1-4,1-6,1-7)$, while for the case $\mathrm{S} 2$ this sequence is: $(2-1,2-3,2-2)$. Drawbacks of the proposed approach to sequence description are clearly visible. For instance, parts 1-3 and 1- 6 should be connected together before their connecting with the rest of the structure, but serial description of the sequence makes this solution impossible.

The method bases on assigning each case part in the database and each part of the structure $\mathrm{S} 0$ to one of predefined classes. The classification process is conducted by an expert. It is a key, and at the same time most problematic, part of the method. We should remember that hull parts are difficult to classify unequivocally. Geometrically similar parts play frequently different roles in the structure and require different treatment in the assembly sequence.

In [20], Qu et al. propose 14 classes of parts, but they do not define them precisely. To analyse the method, we can assume a smaller number of classes due to significant simplification of the analysed structures. The adopted classification of parts is shown in Tab. 1.

Tab. 1. Classification of parts of analysed structures

\begin{tabular}{|c|c|c|c|}
\hline Class of parts & Parts of S1 & Parts of S2 & Parts of S0 \\
\hline A & $1-1$ & $2-1$ & $0-1$ \\
\hline B & $1-2$ & $2-2$ & $0-2$ \\
\hline C & $1-3,1-4$ & - & - \\
\hline D & $1-5$ & - & $0-3$ \\
\hline E & $1-6,1-7$ & - & - \\
\hline F & - & $2-3$ & $0-4,0-5$ \\
\hline
\end{tabular}

According to [20], the structure-case assembly sequences and classification of their parts make the basis for obtaining general rules to be used when creating structure-problem sequences. The case $\mathrm{S} 1$ gives the following sequence of classes: $D \rightarrow B \rightarrow A \rightarrow C \rightarrow E$, while the case S2 gives: $A \rightarrow F \rightarrow B$. 
The inference on the new sequence is performed by determining precedence relations between parts belonging to certain classes. The structure-problem assembly sequence resulting from case $S 1$ is the following: $0-3 \rightarrow 0-2 \rightarrow 0-1$, while that resulting from case $S 2$ is: $0-1 \rightarrow 0-4 \rightarrow 0-5 \rightarrow 0-2$ or equivalent: $0-1 \rightarrow 0-5 \rightarrow 0-4 \rightarrow 0-2$. The following problems can be noticed in the obtained results:

- none of the obtained sequences covers entirely the structure-problem,

- precedence relations obtained based on different structures partially contradict each other $(B \rightarrow A$ and $A \rightarrow B)$.

In [20], the authors suggest complementing the missing information in the obtained sequence with the aid of genetic algorithm. In this process, geometric constraints between parts and the criterion of minimisation of component class changes during serial assembly are taken into consideration. As a result, a sequence is obtained which is realisable in geometric terms and groups homogeneous parts in such a way that they are assembled directly after each other. The sequences obtained for the analysed structure S0 are shown in Fig. 5.

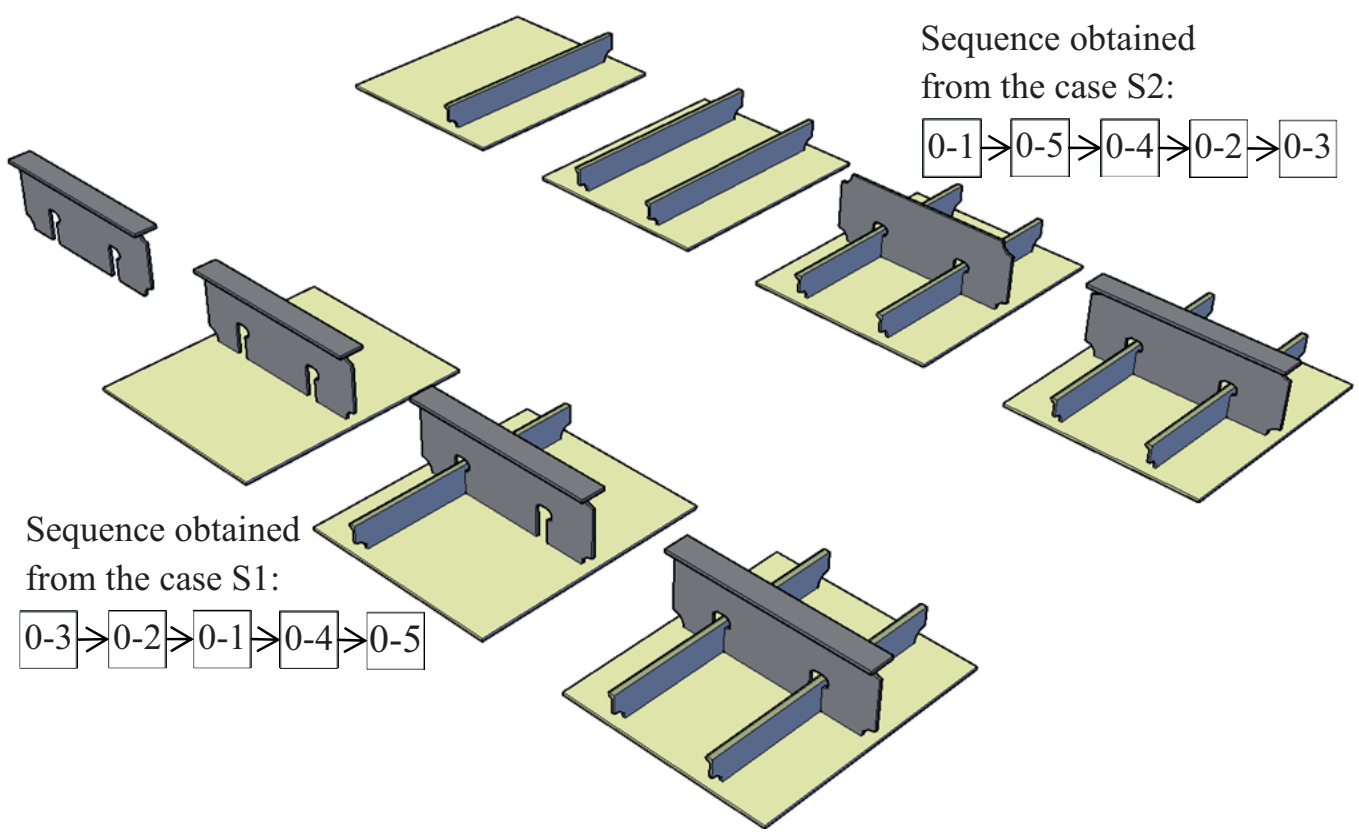

\section{HIERARCHICAL CLUSTERING OF ASSEMBLY UNIT PARTS}

The method described in [21] consists of two parts:

- Hierarchical clustering of assembly unit parts. It makes use of the so-called rule-based expert system and can be used for dividing the structure into subassemblies, with further creation of assembly sequence,

- Optimisation of clustering diversification degree. It makes use of the analytic hierarchy process (AHP) algorithm, but the solution assessment is done solely by an expert and does not contribute much to the problem of assembly sequencing needs.

The proposed method of hierarchical clustering requires defining the fuzzy similarity relationship $u_{i, j} \in[0,1]$ for each pair $(i, j)$ of structure parts. The rules to assess the quantities $u_{i, j}$ are defined in [21]. They are determined by an expert and their use is somewhat problematic, as the expert is expected to plan partially the assembly before assigning the value of $u_{i, j}$. Prior assembly planning refers to positions in which welded joints are to be made. Different values of $u_{i, j}$ are assigned to fillet welds made in bottom, vertical and top direction of connection. However, these positions are determined after determining the order in which some liaisons are done. Thus, this method, on the one hand, makes it possible to divide the structure into subassemblies, but on the other hand, it requires from the expert to define a priori this division.

The authors of [21] also propose common consideration of identical parts which are to be assembled simultaneously or directly after each other. This refers, for instance, to parallel ribs. In the considered case, parts 0-4 and 0-5 can be grouped, which to a certain extent will simplify the analysis as they will be collectively treated as part 0-4.

The adopted values of fuzzy similarity relationships for the analysed structure S0 (Fig. 1) are given in Tab. 2.

Tab. 2. Fuzzy similarity relationships for SO structure

\begin{tabular}{|c|c|c|c|c|c|}
\cline { 3 - 6 } \multicolumn{2}{c|}{} & \multicolumn{4}{|c|}{ part } \\
\cline { 2 - 6 } \multicolumn{2}{c|}{} & $0-1$ & $0-2$ & $0-3$ & $0-4$ \\
\hline \multirow{4}{*}{\begin{tabular}{c}
$\vec{\Xi}$ \\
\multirow{2}{*}{}
\end{tabular}} & $0-1$ & 1 & 0.7 & 0 & 0.8 \\
\cline { 2 - 6 } & $0-2$ & 0.7 & 1 & 0.8 & 0.5 \\
\cline { 2 - 6 } & $0-3$ & 0 & 0.8 & 1 & 0 \\
\cline { 2 - 6 } & $0-4$ & 0.8 & 0.5 & 0 & 1 \\
\hline
\end{tabular}


It was assumed that the plate-rib and plate-T-frame liaisons will be done in bottom position. According to the analysed method, it can be assumed at this stage that the T-frame will also be prefabricated in bottom position, i.e. before welding to the plate and ribs. Prefabricating the T-frame is treated as rib-plate liaison, therefore $u_{2,3}=u_{3,2}=0.8$.

Finally, hierarchical division of parts into groups is obtained, which can be interpreted as subassembly partition of the assembly unit (Fig. 6). The parameter $\lambda \in[0,1]$ represents granularity of the partition: $\lambda=1$ means partition into individual parts, while $\lambda=0$ means the assembly unit as a whole.

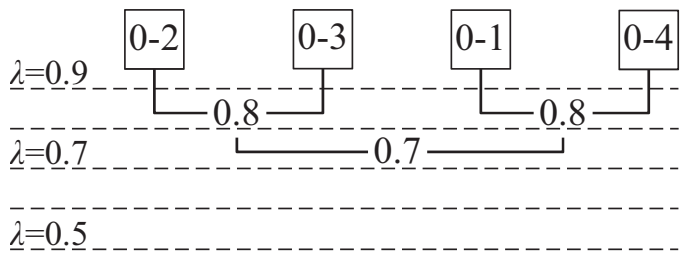

Fig. 6. Clustering tree of assembly unit partition

Partition of the analysed unit at the level of 0.8 enables making distinction between two subassemblies: T-frame, and plate with ribs. This is consistent with the expected assembly sequence, but at a very general level. This way, a lot of relevant information concerning the order of doing liaisons is omitted.

\section{CONCLUSIONS}

The article presents a literature review of assembly planning methods. The review takes into consideration both issues focused on assembly of mechanisms and those dedicated to ship hulls, in an attempt to find common features of these methods and development trends in this branch of science. In the world literature, works on assembly of mechanisms significantly dominate over those dedicated to hulls and marine structures. However, these solutions cannot be easily adapted to hull assembly problems. Therefore, the area od search for the best method was narrowed to methods dedicated to the shipbuilding industry. The analysis omits earlier works by the authors of the present article [8] [22] [23] [24] to avoid possible objections on bias in selections made in Chapter 3.3.

The analysis of ASP methods dedicated to hull structures was performed based on three simplified exemplary structures, two of which constitute "cases" stored in the database while one is the "problem", for which the assembly sequence is to be created. Use was made of three methods described in the literature which are dedicated to different ship hull structures. It is noteworthy that all methods:

- require significant participation of an expert in: describing the assembly unit, classifying parts and/or liaisons, and final supplement of the sequence with missing pieces of information,
- were tested by the authors on cases of small assembly units, for which expert's participation was a problem,

- make use of computational intelligence algorithms, which perform well in discrete problems with large search spaces. Two of the analysed methods are similar to each other, as they make use of the database and the CBR algorithm for generating assembly sequences. A basic difference between them is the method of sequence recording. The performed analysis has revealed that the activities-oriented serial sequence provides opportunities for better mapping of serialparallel assembly. This type of assembly is widely used in contemporary shipbuilding industry.

The third method was not developed to generate the assembly sequence, it was rather dedicated to plan assembly partition of the structure. This function is very close to sequencing, but the final result of hierarchical partition has only the form of a sketch of sequence, as it is oriented on parts.

Based on the performed observations, some assumptions can be formulated with regard to required features and development directions of hull assembly sequence planning methods:

1. The sequence model should be oriented on activities, i.e. doing liaisons, which constitute the main component of hull assembly labour consumption and cost generation.

2. Possibility of serial-parallel planning - it is especially important in the cases of plans for assembly units composed of hundreds of thousands of parts. Serial plans, in general, do not reflect the essence of parallel production of many hull sections and blocks.

3. Limited experts' involvement in the current calculation process, along with computational complexity of algorithms resistant to the effect of search space explosion - these conditions are necessary for the methods to find practical application for larger structures, such as hull sections and blocks.

4. Use of technological knowledge stored in the database of similar structures - the problem of similarity assessment for efficient assembly sequence generation still remains open. Literature examples and the performed analysis name a number of unsolved problems here.

5. Use of hierarchical grouping methods for hierarchical partition of the assembly unit - this approach seems promising as preliminary stage of assembly planning, as it significantly reduces the sequence search space.

6. Use of evolutionary algorithms in the sequence optimisation process, which are very productive in large search spaces with numerous constraints, due to the penalty function. During hull assembly, a number of additional problems can occur which have not been taken into consideration yet in ASP methods:

- welding deformations and assembly usefulness ofsubassemblies,

- stability of subassemblies during welding processes and their potential for rotation and transport,

- safety of work at height and in narrow spaces,

- ergonomics of work in forced welding positions,

- accessibility of liaisons for welding robots, 
- different welding speeds and parameters of welds made in different welding positions,

- synchronisation with hull equipping operations,

- effect of assembly plan on work organization at part machining departments and material management.

The above issues are to be taken into consideration when improving ASP methods dedicated to hulls. This is a serious challenge at both conceptual and efficiency level. It is noteworthy that such a complex problem concerns the assembly unit with gigantic dimensions, not to be found elsewhere.

Wider and wider use of computational intelligence methods, including image processing methods, provides opportunities for automation of processes concerning database development and description of new structures. It is essential for the development of ASP methods to take into consideration aspects of cooperation with CAD/CAM systems which are already in use in design offices. These systems are used for creating full descriptions of parts and liaisons of the entire structure. Moreover, 3D modelling provides opportunities for automatic analysis of geometric conflicts occurring during the assembly. The use of the above information would allow us to reduce significantly experts' participation in ASP processes of ship hulls.

\section{BIBLIOGRAPHY}

1. Yun D. H., Choi S. I., Kim S. H., Ko K. H.: Registration of multi-view point clouds for application to ship fabrication, Graphical Models, Vol.90, pp. 1-12, 2017.

2. Back M. -G., Lee D. -K., Shin J. -G., Woo J. -H.: A study for production simulation model generation system based on data model at a shipyard, International Journal of Naval Architecture and Ocean Engineering, Vol.8(5), pp. 496510, 2016.

3. Zhao-Hui W., Ji-Wang D., Ming-hua Z., Xiu-min F.: Survey on Flexible Shipbuilding Technologies for Curved ShipBlocks, Procedia Engineering, Vol. 174, pp.800-807, 2017.

4. Banerjee S. K.: Shipyard production systems design: a statistical approach, International journal of Production Research, Vol. 6, pp. 541-555, 1979.

5. Cho K.-K., Sun J.-G., Oh J.-S.: An automated welding operation planning system for block assembly in shipbuilding, International Journal of Production Economics, 60-61, pp. 203-209, 1999.

6. Chi Z., Jun S.: Intelligentized work-preparation for ship hull construction with Optimized Assembly Planning System, Proceedings of the IEEE International Conference on Systems, Man and Cybernetics, Istanbul, Turkey, 10-13, pp. 2740-2744, 2010.
7. Ghandi S., Masehian E.: Assembly sequence planning of rigid and flexible parts, Journal of Manufacturing Systems, Vol. 36, pp. 128-146, 2015.

8. Iwańkowicz R.: Methods of sea-going ship hull assembly sequencing and scheduling, Collegiate Publication of the West Pomeranian University of Technology Szczecin, ISBN 978-83-7663-216-2, 2016.

9. Robinson R.W.: Counting labeled acyclic digraphs, in: New directions in the theory of graphs, Ed. F. Harary, [b.m.], Academic Press, 239-273, ISBN-13 978-0123242556, ISBN$10012324255 \mathrm{X}, 1973$.

10. De Fazio T., Whitney D.: Simplified generation of all mechanical assembly sequences, IEEE Journal of Robotics and Automation, Vol. 3(6), pp. 640-658, 1987.

11. Bourjault A.: Contribution a uneapprochemkthodologique de l'assemblage automatis: Elaboration automatique des sequences operatiores, Thèse de doctorat, Université de Franche-Comte, 1984.

12. Homem de Mello, L. S., and Sanderson, A. C.: AND/OR graph representation of assembly plans, IEEE Transactions on Robotics and Automation, Vol. 6, no. 2, pp. 188-199, 1986.

13. Homem de Mello, L. S., and Sanderson, A. C.: Planning repair sequences using the And/Or graph representation of assembly plans, Proceedings of the 1988 IEEE International Conference on Robotics and Automation, Philadelphia, USA, pp. 1861-1862, 1988.

14. Eng T.-H., Ling Z.-K., Olson W., McLean C.: Feature-based assembly modeling and sequence generation, Comput. Ind. Eng., Vol. 36, pp. 17-33, 1999.

15. Voutchkov I., Keane A., Bhaskar A., Olsen T.M.: Weld sequence optimization: the use of surrogate models for solving sequential combinatorial problems, Computer Methods in Applied Mechanics and Engineering, vol. 194, pp. 3535-3551, 2005.

16. Sasaki Y., Sonda M., Ito K.: Development of a computeraided process planning system based on a knowledge base, Journal of Marine Science and Technology, Vol. 7, pp. 175179, 2010.

17. Bonneville F., Perrard C., Henrioud J.M.: A genetic algorithm to generate and evaluate assembly plans, IEEE Symposium on Emerging Technology and Factory Automation, Vol. 2, pp. 231-239, 1995.

18. Chen \& Liu Chen S.F., Liu Y.J.: An adaptive genetic assembly-sequence planner, Int J Comput Integr Manuf, Vol. 14(5), pp. 489-500, 2001. 
19. Seo Y., Sheen D., Kim T.: Block assembly planning in shipbuilding using case-based reasoning. Expert Systems with Applications, Vol. 32, pp. 245-253, 2007.

20. Qu S., Jiang Z., Tao N.: An integrated method for block assembly sequence planning in shipbuilding, The International Journal of Advanced Manufacturing Technology, Vol. 69 (5-8), pp. 1123-1135, 2013.

21. Zhong Y., Xue K., Shi D.: Assembly unit partitioning for hull structure in shipbuilding, Computer- Aided Design, Vol. 45(12), pp. 1630-1638, 2013.

22. Iwańkowicz R.: An efficient evolutionary method of assembly sequence planning for shipbuilding industry, Assembly Automation, Vol. 36(1), pp. 60-71, 2016.

23. Iwańkowicz R.: Optimization of assembly plan for large offshore structures, Advances in Science and Technology Research Journal, Vol. 6(16), pp. 31-36, 2012.

24. Iwańkowicz R., Taraska M.: Self-classification of assembly database using evolutionary method, Assembly Automation, Vol. 38(1), 2017.

\title{
CONTACT WITH THE AUTHORS
}

\author{
Urbański Tomasz \\ Graczyk Tadeusz \\ Taraska Michał \\ e-mail:michal.taraska@zut.edu.pl
}

West Pomeranian University of Technology in Szczecin

Faculty of Maritime Technology and Transport
Al. Piastów 41
70-065 Szczecin
Poland

Remigiusz Iwańkowicz

Maritime University of Szczecin

Faculty of Transport Engineering and Economics

1-2 Wały Chrobrego St.

70-500 Szczecin

Poland 